\title{
Dasselbe nochmal: Narratives Potenzial von Bildwiederholungen in frühneuzeitlichen Romanen
}

\begin{abstract}
Illustrated novels are characterized by the material co-existence of written text and images. This combination of figuration and writing allowing intersemiotic interpretation reached new heights with the technical advances of the turn of the sixteenth century. The multiple use of the same image within one illustrated narrative is a little-studied phenomenon linked to this special type of text. Up to now, research has mainly concentrated on the historical conditions of production to explain these repetitions. One could, however, also ask what the narrative consequences of such multiple uses of identical illustrations are. In this contribution, an attempt is made to partially answer this question by means of a close reading of a small extract of Georg Wickram's Ritter Galmy (1539), a typical example of duplication within one novel.
\end{abstract}

Illustrierte Romane zeichnen sich durch die materiale Gleichzeitigkeit von Bild und Schrift, d. h. durch eine bisemiotische Konfiguration der Buchseite, aus, die aufgrund technischer Innovation erst an der Schwelle vom 15. zum 16. Jahrhundert eine Hochkonjunktur erfährt. Durch sie werden intersemiotische Interpretationsspielräume eröffnet, für die die Wechselbeziehung der unterschiedlichen Zeichensysteme konstitutiv ist. Die mehrfache Verwendung identischer Bilder innerhalb eines Romans stellt dabei ein kaum untersuchtes Phänomen dar, das primär als Ausdruck marktwirtschaftlichen Kalküls verstanden wird. Stattdessen stehen im Zentrum der folgenden Überlegungen die narrativen Konsequenzen solcher Bildwiederholungen, die anhand eines Close-Readings eines knappen Auszugs aus Georg Wickrams Ritter Galmy (1539) exemplarisch vorgeführt werden.

Auch wenn uns die Gleichzeitigkeit von Bild und Schrift in einem Artefakt heute in unterschiedlichen literarischen und nicht-literarischen Kontexten vertraut oder gar als Standardfall erscheint (man denke z. B. an Printwerbung oder generische Schrift-Bild-Formen wie den Comic), ${ }^{1}$ so handelt es sich dabei am Übergang vom 15. zum 16. Jahrhundert doch um eine Konfiguration, die

1 So proklamiert Susanne Blazejewski gar: „Die Kombination von Wort und Bild stellt heute in allen Medien den Normalfall dar.“ (Susanne Blazejewski, Bild und Text - Photographie in autobiographischer Literatur: Marguerite Duras' 'L'amant' und Michael Ondaatjes 'Running in the Family'. Würzburg 2002, S. 14; Hervorhebung NP).

Nicolas Potysch, Ruhr-Universität Bochum

Ә Open Access. ( 2019 Nicolas Potysch, published by De Gruyter. (cc) BY-NC-ND This work is licensed under a Creative Commons Attribution-NonCommercial-NoDerivatives 4.0 International License. https://doi.org/10.1515/9783110563016-010 
aufgrund technischer Innovation für den literarischen Markt gerade erst attraktiv geworden war. Diese Attraktivität war in erster Linie eine wirtschaftliche, bei der die Rentabilität der verantwortlichen Offizin und die gesicherte finanzielle Situation der mit der Buchproduktion verbundenen Akteure (Autor, Setzer, Reißer, Schneider, Lektor, Drucker, etc.) im Zentrum stand. Darüber hinaus stellt die Entwicklung effizienter Drucktechniken und -maschinen aber auch im Sinne einer Schlüsseltechnologie die ,Bedingung der Möglichkeit' für die Herausbildung literarischer Genres dar, ${ }^{2}$ deren genuines Merkmal die semiotische Verschränkung von Schrift und Bild ist. Wenngleich diese Korrelation bereits verschiedentlich ausführlich beschrieben wurde, ${ }^{3}$ so existieren doch bis dato kaum beachtete Phänomene, die sich ausgerechnet in dieser von Experimenten und Unwägbarkeiten bestimmten Sondierungsphase moderner Buchproduktion herausgebildet haben und denen bis in die Gegenwart hinein systematische Relevanz zukommt. Eines dieser Phänomene wird im Folgenden mitsamt dem damit verbundenen narrativen Potenzial näher differenziert und analysiert: die Bildreplik, d. h. die intratextuelle weil romaninterne Mehrfachverwendung einer identischen Bildtextur. ${ }^{4}$

Ich beginne meine Argumentation mit der Darlegung des exemplarischen, historischen Gegenstands und des Phänomens, wobei ich an geeigneter Stelle abbrechen werde. ${ }^{5}$ Derart skizziere ich die Herausforderung, die Bildwiederholungen

2 Eine Technologie ist dann eine Schlüsseltechnologie, wenn durch sie zugleich die technischen Möglichkeiten einer Gesellschaft geradezu sprunghaft ansteigen und eine gesellschaftliche Entwicklung beschleunigt oder überhaupt erst ermöglicht wird. Vgl. dazu Mae Keary, 'Key Technologies - Chasing the Gap'. In: Aslib Proceedings 43/5 (1991), S. 161-172.

3 Vgl. dazu beispielsweise das formal und nicht inhaltlich gedachte Genre des ,Bildbuchs،, das Knape als „feste[s] und verbreitete[s] Prinzip der Buchgestaltung im Bereich der Moralistik und Satire“ (S. 153) bezeichnet. Vgl. Joachim Knape, 'Mnemonik, Bildbuch und Emblematik im Zeitalter Sebastian Brants (Brant, Schwarzenberg, Alciati)'. In: Mnemosyne. Festschrift für Manfred Lurker zum 60. Geburtstag. Hg. v. Werner Bies und Hermann Jung. Baden-Baden 1988, S. $133-178$.

4 Zum systematischen Anspruch, Bilder als Texte bzw. Texturen zu beschreiben, der sich maßgeblich aus der Semiotik speist vgl. z. B. Hartmut Stöckl, Die Sprache im Bild, das Bild in der Sprache: Zur Verknüpfung von Sprache und Bild im massenmedialen Text. Konzepte, Theorien, Analysemethoden. Berlin, New York 2004; Thomas M. Susanka, Foto/grafie. Zur Rhetorik von Medium und Bild. Mit einer Fallstudie zu James Nachtwey. Berlin 2015; Ulla Fix und Hans Wellmann, 'Sprachtexte - Bildtexte. Bemerkungen zum Symposion Bild im Text - Text und Bild vom 6.-8. April 2000 in Leipzig’. In: Bild im Text, Text im Bild. Hg. v. Ulla Fix und Hans Wellmann. Heidelberg 2000, S. XI-XVII.

5 Ausführlicher widme ich mich dieser Thematik anhand weiterer Beispiele - aus dem Fortunatus (1509), Georg Wickrams Ritter Galmy (1539), Georg Messerschmidts Brissonetus (1559), Thüring von Ringoltingens Melusine (1587) in der Fassung des Buchs der Liebe und Karl Arnold 
innerhalb des Lektüreprozesses für die Intersemiose - also die das einzelne Zeichensystem überschreitende Bedeutungszuweisung von Schrift und Bild - und die Konstruktion einer global sinnkonstanten, erzählten Welt darstellen.

\section{Bildwiederholungen im Ritter Galmy I}

Der 1539 in Straßburg bei Jacob Frölich veröffentlichte und vermutlich von Georg Wickram verfasste Roman Ein schoene und liebliche History von dem edlen und theüren Ritter Galmien berichtet vom Aufstieg des jungen Ritters Galmy am Hofe des bretonischen Herzogs. ${ }^{6}$ Der Ritter, der bald in höfischer Minne zur Frau seines Lehnsherrn entbrennt, ist trotz seines ritterlichen Erfolges nach einer Intrige gezwungen, ins Exil in die Dienste des schottischen Königs $\mathrm{zu}$ fliehen. Lediglich um seine verehrte Herzogin zu schützen, kehrt er als Mönch verkleidet in die Bretagne zurück und gewinnt für sie einen Gerichtskampf, ein Gottesurteil, durch das ihre moralische Unversehrtheit bewiesen wird. Nachdem der bretonische Herzog bald darauf an einer Krankheit verstirbt, kann Galmy aus dem Exil zurückkehren, die Herzogin wenig später ehelichen und selber zum Herzog der Bretagne erhoben werden. Bei der Lektüre des Romans begegnen insgesamt 61 Bilder, ${ }^{7}$ denen jedoch nur 37 Druckstöcke zugrunde liegen. Die Ursache für diese Diskrepanz erklärt sich dadurch,

Kortums Jobsiade (1824) - in Wiederholt doppeldeutig in Bild und Schrift - Ambiguität im durchbilderten Roman. Hannover 2018.

6 Als eines der Zentren der Reformation kommt Straßburg auch als deutsch-französischer Kontaktzone im 16. Jahrhundert maßgeblicher Einfluss auf den Buchmarkt und das Druckergewerbe der Zeit zu. Josef Benzig geht dabei so weit, superlativisch zu betonen: „L'importance de Strasbourg pour l'histoire de l'imprimerie n'est plus à démontrer“ (Josef Benzig, Bibliographie Strasbourgeoise. Bd. 1. Baden-Baden 1981, S. 5). Unabhängig davon, ob diese Einschätzung uneingeschränkt zu teilen ist, konnte Straßburg daher wohl bereits in der Frühzeit des erstarkenden, ortsfesten, deutschen Buchmarkts auf eine handwerkliche Tradition sowie auf ein ausgeprägtes Selbstverständnis und -bewusstsein der dazugehörigen Berufsstände zurückblicken. Damit ein Buch wie der Ritter Galmy in diesem wirtschaftlichen Umfeld zum Bestseller werden konnte - Gotzkowsky verzeichnet alleine für das 16. Jh. 13 Ausgaben -, musste es sich entweder durch einen besonders günstigen Preis oder eine hohe Qualität gegenüber dem breiten Konkurrenzangebot auszeichnen. Vgl. Bodo Gotzkowsky, 'Volksbücher'. Prosaromane, Renaissancenovellen, Versdichtungen und Schwankbücher. Bibliographie der Deutschen Drucke. Teil I: Drucke des 15. und 16. Jahrhunderts. Baden-Baden 1991, S. 443-448. [Vgl. für die Bedeutung von Straßburg als Druckzentrum auch den Beitrag von Bertelsmeier-Kierst in diesem Band (Seite 37).]

7 Den verbreiteten Ausdruck Illustration vermeide ich trotz seiner erhellenden Etymologie konsequent, da mir mit seiner Verwendung sowohl im deutsch- als auch englischsprachigen 
dass innerhalb des Romans acht Bilder zweimal, fünf dreimal, zwei viermal und nur die übrigen 22 singulär dargeboten werden. Die Bildtexturen sind von einer Ausnahme abgesehen - immer ungefähr am Kapitelanfang gesetzt, jedoch beziehen sie sich nicht zwingend auf die dort unmittelbar beschriebenen Ereignisse. So es die Seitenaufteilung erlaubte, wurde offenbar versucht, die Bilder unmittelbar unter oder innerhalb der Kapitelüberschrift einzufügen. Der sorgfältige Satz und die hohe Qualität des im Oktav-Format vorliegenden Romans fallen auf und lassen auf eine sorgfältige Produktion und nennenswerte Fertigungskosten schließen. Mit Ausnahme des quadratischen Titelbildes sind sämtliche Bilder als leichte Querrechtecke (Verhältnis 7:8) geboten, wobei jedes Bild erneut von einer kräftigen schwarzen Linie gerahmt wird. Während die Bildtextur auf der Titelseite ca. zwei Drittel des Satzspiegels einnimmt, kommt den anderen Bildern etwa drei Fünftel der bedruckten Seitenfläche zu.

Da offensichtlich weniger Holzschnitte angefertigt wurden, als Kapitel vorhanden sind, liegt möglicherweise zunächst der Gedanke nahe, die Wiederholungen dadurch zu erklären, dass Frölich bzw. Wickram dem Leser für jedes Kapitel ein Leitbild bieten wollte und so gezwungen war zu wiederholen. Aufgrund zweier Überlegungen ist diese Annahme jedoch wenig plausibel. Erstens verfügt nicht jedes Kapitel über genau ein Bild: Im 54. Kapitel, das mit fünfeinhalb Seiten keineswegs besonders umfangreich ist, finden sich anders als in den sonstigen Kapiteln zwei Bildtexturen, von denen außerdem keine mehrfach gebraucht wird. Zweitens wäre das Bildprogramm des Romans leicht für eine solche Situation anzupassen gewesen - die Offizine verfügten in der Regel über einen großen Bestand an Druckstöcken. Außerdem wird begründet gemutmaßt, dass Wickram nicht nur den Wortlaut des Ritter Galmy, sondern auch die dazugehörigen Bilder genauestens plante. ${ }^{8}$ Wäre die obige These zutreffend, so hätte das Verhältnis von Bildtextur zu Kapitel auch über die Kapitelanzahl oder weitere Holzschnitte reguliert werden können.

Bei der Lektüre des Romans begegnet auf fol. $9^{\mathrm{r}}$ ein Bild (s. Abb. 1), das eine intime Szenerie zeigt: Eine Frau, die ihrer prunkvollen Kleidung nach wohl von edlem Stande ist, steht am Bett eines offensichtlich erkrankten oder sterbenden

\footnotetext{
Raum eine implizite und explizite Aufwertung des Illustrierten, d. h. der Schrift und eine Abwertung des Illustrierenden, den Bildern, verbunden scheint.

8 Vgl. Peter Schmidt, 'Literat und "selbstgewachsener Moler”. Jörg Wickram und der illustrierte Roman der Frühen Neuzeit'. In: Künstler und Literat. Schrift- und Buchkultur in der europäischen Renaissance. Hg. v. Bodo Guthmüller, Berndt Hamm und Andreas Tönnesmann. Wiesbaden 2006, S. 143-194.
} 
Mannes und leistet diesem Beistand. Sie hat seine rechte Hand mit ihrer gefasst und sieht den Kranken unverwandt an. Am rechten Bildrand steht eine weitere Frau - ihrem Gewand nach wohl eine Zofe - die ebenfalls den Kranken betrachtet. Die Identifikation der dargestellten Figuren innerhalb der Romanepisode ist in diesem Fall unproblematisch bereits anhand der Kapitelüberschrift möglich: „Wie die Hertzogin mit jren Junckfrawē den Ritter Galmien heymsuochet / jn auff seinem betth ligen findet / Und wie der Ritter von der Hertzogin gedroest ward“ (fol. $8^{\mathrm{v}}$, Hervorhebungen NP]).

Die Herzogin betritt somit in standesgemäßer und anständiger Begleitung „jre[r] Junckfrawē“ (fol. $8^{\mathrm{v}}$ ) das Gemach des Ritters Galmy. Dort spricht sie mit dem leidenden, im Bett liegenden Ritter - der dann auch der Männergestalt im Bild zugeordnet werden kann - und vergewissert sich, dass die Botschaft von der Minnekrankheit des Ritters, die Galmys Freund Friderich ihr übermittelte, der Wahrheit entspricht. Das anfängliche Zögern des Ritters, sich seiner Herrin zu offenbaren, überwindet die Herzogin dadurch, dass sie „zuohandt die beyden junckfrawen / mit sampt deß Ritters knaben / in jr gemach schicken theht“ (fol. $9^{r}$ ), nicht ohne jedoch explizit zu betonen: „Dieweil ich doch verstanden hab / du [=Galmy] mich nicht anderst / dann in züchten und eeren liebhabest / und allein meines drostes begeren thuost“ (fol. 10 ${ }^{\mathrm{v}}$ ).

Nach einem längeren Dialog, in dem Galmy der Herzogin seine Liebe gesteht, überreicht sie ihm - relativ unvermittelt - einen Ring als Liebesgabe. Mittels dieses „zeychen warer unn rechter liebe“ (fol. $11^{\mathrm{v}}$ ) schließt sie so einen Treuebund mit ihrem Ritter. Besondere Aufmerksamkeit legt die Schrift-Bild-Textur dabei auf das Motiv der ,Hand'. Galmy berichtet seiner Geliebten, wie er sich vor kurzem auf einem Jagdausflug in sie verliebt hat: „[S]o bald aber ewer schoene weisse hand / inn die mein verschlossen ward / augenblicklich mich ein brinnender flamn umb mein herz entzünden thet / und von solchen tag an / die liebe sich in mir staetigs gemeret / und so krefftigklich zuogenumnen“ (fol. 11v). Im Kontext dieser Aussage erhält die Tatsache, dass das Bild die Herzogin just in dem Moment zeigt, als sie dem Ritter erneut „ir schneeweisse hand bieten thet“ (fol. $11^{v}$ ), zusätzliche Brisanz - entzündete diese Geste doch die Liebe des Ritters. Der Krankenbesuch der Herzogin ist schließlich von Erfolg gekrönt: Der Ritter gesundet durch die Fürsorge seiner Herrin. Die Medizin, die der Bildbetrachter in der dargestellten Glasphiole der Zofe vermuten mag, findet sich dabei schriftlich als „koestlich confeckt unn latwergen“ (fol. $12^{\mathrm{r}}$ ) wieder. Wie genau der Leser sich allerdings eine Medizin für einen liebeskranken Ritter vorzustellen hat, wird nicht verraten, doch deutet es der körperliche Kontakt zwischen Ritter und Herzogin im zuvor erotisch aufgeladenen Händereichen und der pointierten Hervorhebung, dass die Herzogin sich ,aller einig bey dem Ritter“ (fol. 9 ${ }^{\text {r }}$ 


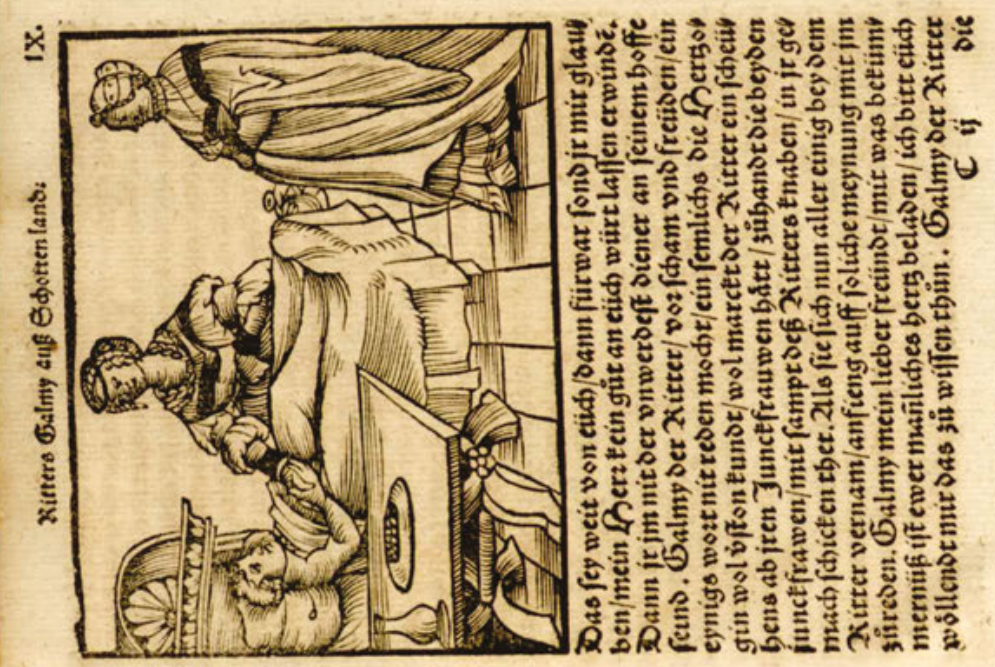

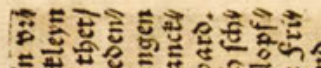

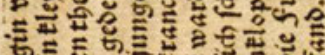

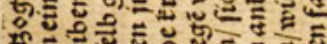

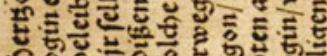
₹ C्व

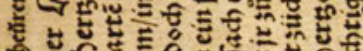

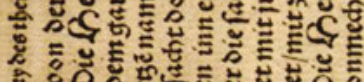

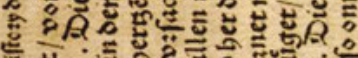
c훙

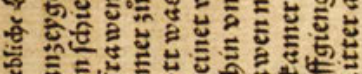

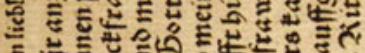

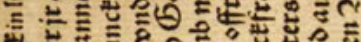
ษั

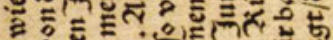

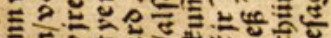
츤


눌을
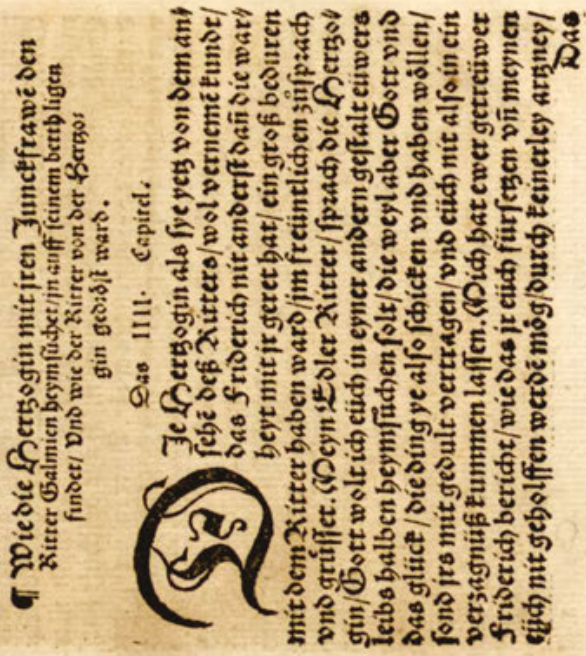

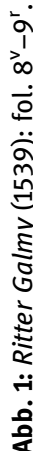


befand, an. Zudem endet die intime Zweisamkeit der beiden Liebenden mit der erneuten Betonung des erotischen Moments:

Die zeit aber kam / das die Junckfrawen jrem befelch nach / schier kummen sollten / die Hertzogin guot bedunckt / ein abscheyd von dem Ritter zuo nemmen / also sprach. Mein aller liebster Galmy / uns wil nit lenger gezymmen / bey einander zuo bleiben / deßhalben ich ein freündlich urlob von dir beger / im ihr schneweisse hand bieten thet. (fol. 11 ${ }^{\mathrm{v}}$ )

Im Fortlauf der Ereignisse steigt Galmy erst weiter am herzoglichen Hofe auf, indem er sich und seinem Lehnsherren Ruhm und Anerkennung auf einer Vielzahl von Turnieren erwirbt, bevor er dann aufgrund einer erpresserischen Intrige, in deren Zentrum seine Minne zur Herzogin steht, ins Exil nach Schottland flüchten muss. Nur für einen gerichtlichen Zweikampf kehrt er verkleidet zurück und verhindert so, dass eine weitere Verschwörung seine Herrin auf den Scheiterhaufen bringt.

Wenn dann auf fol. $134^{\mathrm{r}}$ (s. Abb. 2) das Bild von fol. $9^{\mathrm{r}}$ erneut begegnet, stellt dies den Leser bzw. die Leserin vor ein Dilemma: Die Bedeutung, die dem Bild eingangs des Romans im vierten Kapitel zugewiesen wurde - Herzogin und Galmy im Zwiegespräch im Gemach des Ritters - ist hier innerhalb der erzählten Welt unmöglich, da Galmy nach wie vor in Schottland weilt und aktuell von seinem Vertrauten Friderich besucht wird.

Stattdessen legen der folgende Passus und das erneut dargebotene Bild eine andere Krankenbett-Situation nahe:

Nun begab sich in der zeit / die weil Friderich inn Schottenland was / das der Hertzog inn ein schwere kranckheyt fiel / Darvon ein neüwes leydt inn gantzem Britanien endtston thett. Als aber vil mit jm versuochet / unnd als umb sunst was. Der Hertzog zuo letst von diser welt verschied. (fol. 135 $5^{\mathrm{r}}$ )

Der Herzog ist plötzlich sehr schwer erkrankt, die Ärzte können ihm nicht mehr helfen und so stirbt er bzw. liegt in der Momentaufnahme des Bildes im Sterben. Ihm kommt daher in der Bildtextur wohl die Position zu, die zuvor der kranke, im Bett liegende Galmy eingenommen hat. Die Herzogin hält auch seine Hand und spendet ihm Trost. Eine Hofdame mit einer Phiole in der Hand nimmt die Position der „vil mit jm versouchenden“ Ärzte ein.

Aber wer wird durch die Bildtextur nun im Bett liegend vor Augen geführt Galmy oder der Herzog? Handelt es sich um eine Sterbe- oder eine Genesungsszene? Diese durch die Wiederholung induzierten Irritationen gilt es zu verarbeiten. Bevor ich hier nun mit meiner Auslegung des Ritter Galmy fortfahre, 


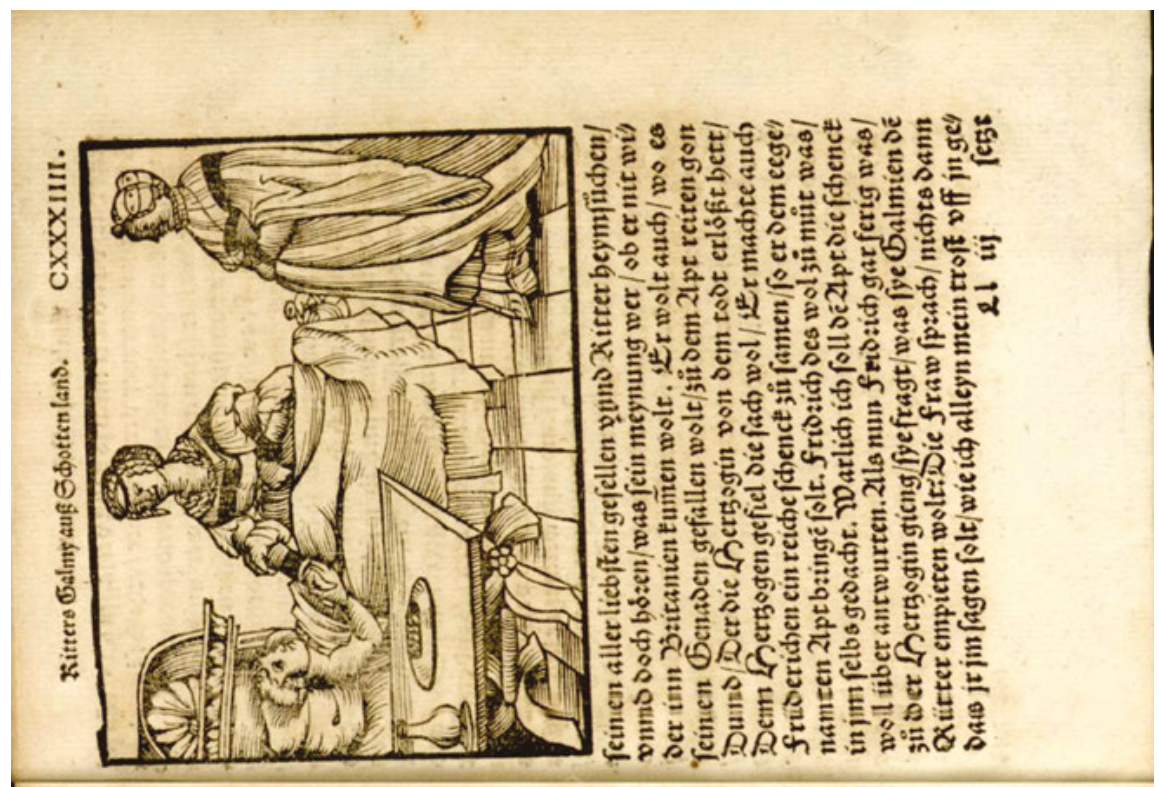

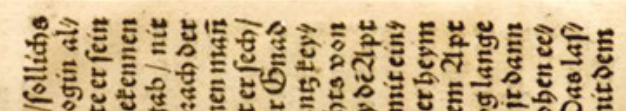
ह

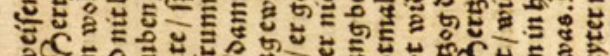
E औ 屯

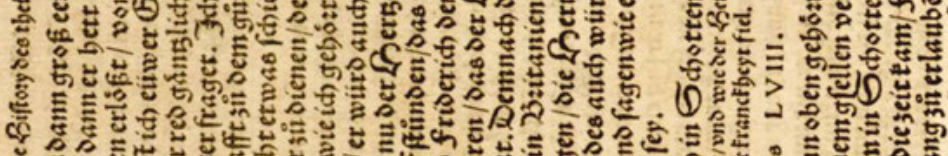

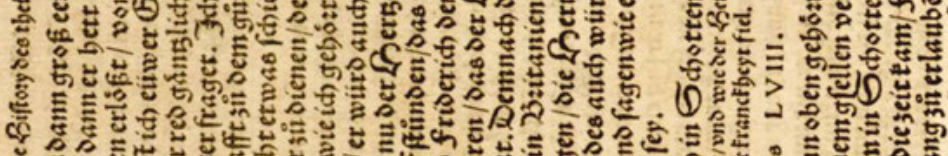

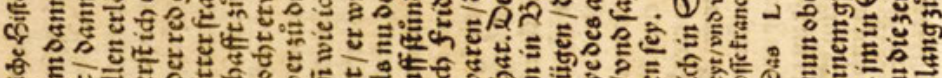

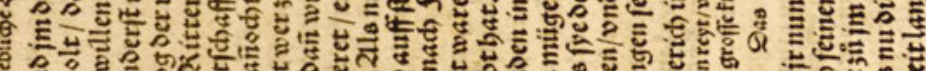

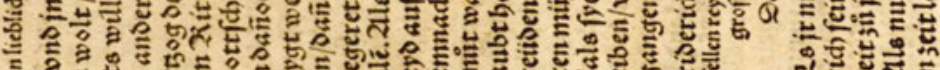

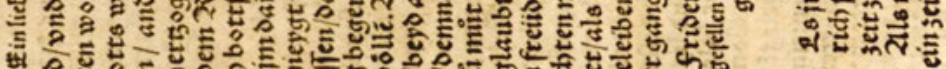

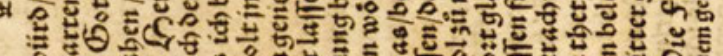

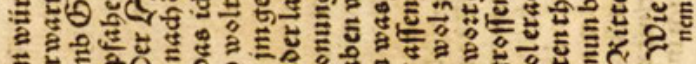

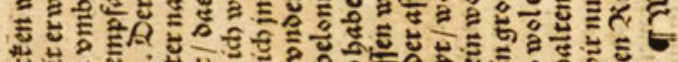

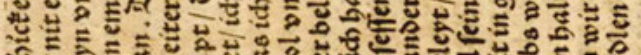

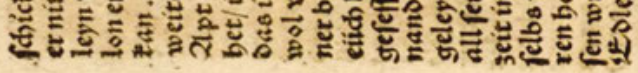

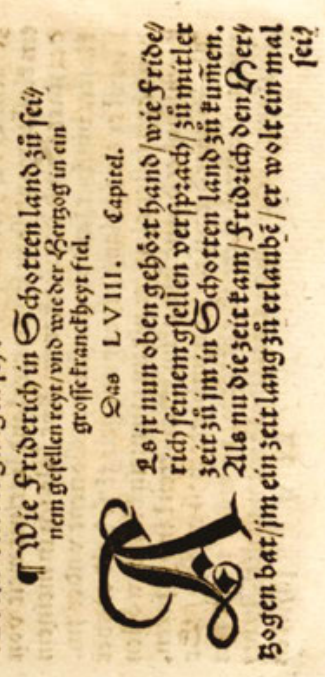


trete ich daher einen Schritt von dieser Einzelinterpretation zurück und konzentriere mich auf das Wechselspiel von Wiederholung und Irritation.

In jüngerer Zeit ist ein erstarktes Interesse an der Durchbilderung der Werke Wickrams (primär) seitens der mediävistischen Forschung zu beobachten. ${ }^{9}$ Die Fragestellungen folgen dabei zwar einem materialphilologisch sensibilisierten Bewusstsein für die Bedeutsamkeit der Korporalität und Materialität der spezifischen Gegenstände, sind jedoch in vielen Fällen noch immer stark auf die von der Schrift diktierte Linearität der Ereignisabfolge fokussiert, die seitens der Bildtexturen dann nur noch unterstrichen bzw. unterstützt wird - Subvention statt Kooperation. Derart entstehen Scheuklappen, die den Blick zwar für einzelne Merkmale der Textur schärfen, gleichzeitig aber andere aus dem Gesichtsfeld verdrängen. So verzeichnet die editionsphilologische Ausgabe der Werke Wickrams von Bolte und Scheel (1901) beispielsweise zwar die Tatsache der Durchbilderung, ${ }^{10}$ reproduziert jedoch keine einzige der Bildtexturen und erwähnt auch die mehrfache Verwendung derselben nicht. Erstmals deskriptiv erfasst ist die multiple Bildwiederholung bei Bodo Gotzkowsky, wenngleich die numerischen Angaben fehlerhaft sind. ${ }^{11}$ Peter Schmidt und Hubertus Fischer fordern schließlich, dass die poetologische Bedeutung und die narrative Funktion, die den Bildtexturen in Wickrams Werken zukommt, zu untersuchen seien. ${ }^{12}$

Eine exemplarische Arbeit, die eine programmatische Veränderung im Umgang mit Wickrams durchbilderten Romanen markiert, stellt Rafael Kuchs „Intermediales Erzählen im frühneuzeitlichen illustrierten Roman. $\mathrm{Zu}$ Struktur und Wirkung der Medienkombination bei Jörg Wickram“ dar. Auch bei Kuch finden

9 Vgl. u. a. Dieter Kartschoke: 'Ritter Galmy uß Schottenland und Jörg Wickram aus Colmar'. In: Daphnis 31 (2002), S. 469-489; oder Raphael Kuch, Intermediales Erzählen im frühneuzeitlichen illustrierten Roman. Köln, Weimar, Wien 2014. Eine ausführliche Übersicht findet sich bei Catherine Drittenbass und André Schnyder (Hgg.), Eulenspiegel trifft Melusine. Der frühneuhochdeutsche Prosaroman im Licht neuer Forschungen und Methoden. Amsterdam, New York 2010.

10 Johannes Bolte und Willy Scheel (Hgg.), Georg Wickrams Werke. Erster Band. Galmy - Gabriotto. Bd. CCXXII. Tübingen 1901 (Bibliothek des literarischen Vereins in Stuttgart), S. XX-XI.

11 Vgl. Gotzkowsky (vgl. Anm. 6), S. 443; und Bodo Gotzkowsky, Die Buchholzschnitte Hans Brosamers zu den Frankfurter 'Volksbuch'-Ausgaben und ihre Wiederverwendungen. BadenBaden 2002, S. 77.

12 Vgl. Schmidt (vgl. Anm. 8) und Hubertus Fischer: 'Wickrams Bilderwelt. Vorläufige Bemerkungen'. In: Vergessene Texte - Verstellte Blicke. Neue Perspektiven der Wickram-Forschung. Hg. v. Maria Elisabeth Müller und Michael Mecklenburg. Frankfurt am Main 2007, S. 199-214. [Zum Einfluss von Bildern auf die Interpretation eines Werkes siehe auch den Beitrag von Frick in diesem Band, S. 254-267.] 
sich Ansätze dazu, die Narration nicht als Schriftgeschichte unterstützt durch bildliche Hinzugaben, sondern als Schrift-Bild-Geschichte $\mathrm{zu}$ verstehen. Dabei fällt jedoch auf, dass Kuch wenig auf systematische Aspekte, wie die theoretischen Grundlagen und die erforderliche Modellierung eines intersemiotischen Narrativs, eingeht. Anders als in den Vorarbeiten und Werkausgaben des (frühen) 20. Jahrhunderts zu Georg Wickram erfährt die 1539er Ausgabe des Ritter Galmy bei Kuch eine detaillierte Beschreibung, bei der auch das Durchbilderungsprogramm inklusive der Bildwiederholungen erfasst wird. ${ }^{13}$ Insbesondere die folgende Hypothese erscheint vor den bisherigen Überlegungen anschlussfähig: „Dabei [= in der Rezeption der Bildwiederholung] schwingt die ursprüngliche Semantik der Bilddarstellung zwangsläufig folienartig mit und regt zur relationalen Verknüpfung mit der neuen Semantik an“. ${ }^{14}$ - Hier wird Ambiguität beschrieben, ohne dann allerdings aus diesem Nebeneinander der Semantiken einen interpretativen Mehrwert abzuleiten. Ohne die notwendigen semiotischen und textuellen Grundlagen wird die Wiederholung von der Schriftextur aus gedacht und an diese angepasst. Friktionen, Kontraste oder Irritationserfahrungen finden dabei kaum Beachtung. Die Wiederholung, so scheint es, hat primär Konsequenzen für das Bild selbst - Kuch spricht sogar von einer „durch die wiederholte Verwendung des Druckstocks erzeugte[n] Ambiguität der Bilderzählung“. ${ }^{15}$

Dass diese Ambiguität aber auch auf Ebene des Schrift-Bild-Textes Konsequenzen hat oder gerade erst auf ihr verortet ist, bleibt hier unberücksichtigt. Gerade für die Auseinandersetzung mit dem Ritter Galmy stellt Kuchs Arbeit daher einerseits einen eindrücklichen Vorläufer und andererseits jedoch eine deutliche Kontrastfolie dar, liegen bei ihm die Deutungs- und Erzählmacht letztlich dann doch beim Schrifttext:

Gemäß den Beteuerungen des Erzählers und der Figuren handelt es sich dabei um eine keusche Liebe, die an sich nicht im Widerspruch zur Beziehung der Herzogin zu ihrem Gatten steht. Dennoch birgt sie die ständige Gefahr missverstanden zu werden und muss daher unbedingt geheim gehalten werden. ${ }^{16}$

Für diese Keuschheit der Liebe, die - wie ich im Folgenden zeigen werde - im Zentrum der von mir vorgestellten Romanepisode steht, finden sich ausreichend Belege innerhalb des Schrifttexts. Die Logik der Erzählung würde also nahelegen, dass die keusche Minne zwar als sexuelle Liebe missverstanden werden kann, letztlich aber moralisch integer bleibt. Dass dem nun möglicherweise gar nicht

13 Vgl. Kuch (Anm. 9), S. 46-96.

14 Kuch (Anm. 9), S. 234.

15 Kuch (Anm. 9), S. 115.

16 Kuch (Anm. 9), S. 47. 
so ist - und das nicht nur, weil der Erzähler und die beteiligten Figuren ständig die moralische Integrität beteuern - werde ich im letzten Teil der Argumentation im Detail zeigen. Mittels der Bildwiederholung - und dem mit ihr einhergehenden unklaren Status des Bildes als Pro- oder Analepse - wird eine zusätzliche Stimme in das Erzählgeflecht des Romans eingewoben, die in der Schrifttextur so überhaupt nicht vorhanden ist. Um diese Lesart $\mathrm{zu}$ plausibilisieren und die narrative Funktion der Bildwiederholung hervorzuheben, trete ich nun den bereits angesprochenen Schritt vom konkreten Gegenstand zurück und frage nach den Mechanismen und Strukturen, die für den präsentierten Romanauszug auf einer systematischen Ebene relevant sind.

\section{Ambiguität, Sinnkonstanz und epistemische Gewissheit}

Auf einer systematischen Ebene bedeutet Ambiguität zu erfahren, nicht (länger) sicher sein zu können, wofür etwas oder wie etwas in Relation zu etwas anderem steht. Im ambigen Fall liegen folglich stets mindestens zwei konkurrierende, ähnlich plausible (Be-)Deutungsvarianten von bzw. in Bezug auf etwas vor. Dieses etwas kann dabei als Ausspruch, als Schrift, Bild, Geste oder Ton, kurz: in verschiedenen semiotischen Systemen begegnen. In dem Moment, wenn sich aufgrund weiterer Informationen Gewissheit einstellt, wie eine Struktur zu deuten ist, endet dieser Zustand - diese kognitive Patt-Situation ${ }^{17}$ - vielleicht sogar unbemerkt. Das literarische Potenzial, das dieses Phänomen für Erzähltexte aus Schrift und Bild - und die damit verbundenen Intersemiose - darstellt, ist vielgestaltig und von unterschiedlicher Komplexität. Daher werde ich mich in den folgenden Überlegungen insbesondere auf die narrative Ebene beschränken, wobei ein materialphilologischer Zugang - also eine Konzentration auf die konkrete Gestalt des Untersuchungsgegenstands anstelle einer Rekonstruktion der Produktionsumstände oder -intentionen - das Fundament bilden wird.

Der Wunsch nach eindeutiger Sinnzuweisung und epistemischer Gewissheit markiert eine anthropologische Präferenz. Als sinngenerierende Subjekte sind Rezipienten bereit, im Umgang insbesondere mit literarischen Texten ko-produzierend tätig zu werden. Das Wissen darum, dass Schrift- und Bildtextur in dieser Form nicht kontingent sind, sondern in der Regel das Resultat von einer

17 Claudia Pinkas, Der phantastische Film. Instabile Narration und die Narration der Instabilität. Berlin, New York 2010, S. 39. 
bis vielen Produktionsentscheidungen sowie der damit verbundenen bewussten Freigabe darstellen, fundiert diese Bereitschaft. Pointiert lässt sich somit festhalten: Dadurch, dass Rezipienten davon ausgehen, dass sich Bild und Schrift aufeinander beziehen bzw. in einer - wie auch immer gearteten - Relation zu einander stehen, ist ihr Verhalten auf die Identifikation möglicher Konnektoren bzw. Verweise von einem System auf das andere hin sensibilisiert. Sie nehmen eine semantische Kohärenz der Gesamttextur (auf globaler Ebene) an, die letztlich unabhängig davon ist, welche semiotischen Ressourcen ihr zugrunde liegen. ${ }^{18}$ Es scheint somit weitaus schwieriger zu sein, Schrift und Bild in einem Buch derart zu kombinieren, dass sich zwischen den beiden Texturen - auch trotz der (Bedeutungs-)Anreicherung durch den Rezipienten - keine plausiblen Sinnbezüge herstellen lassen.

Mittels salienter Strukturen und der dadurch möglichen Markierung von (Einzel-)Elementen kann Einfluss auf den für die Interpretation fundamentalen Prozess der Inferenzbildung genommen werden. ${ }^{19}$ Dadurch, dass bestimmte inhaltliche oder formale Elemente aus der sie umgebenden Ko-Textur herausstechen, wird ihr Stellenwert für den Rezeptionsprozess und die Bedeutungsgenese prominenter. Texturen lassen sich aus dieser Perspektive als komplexe Salienzgeflechte beschreiben, für deren Faktur in der Forschung bereits mehrfach die Metapher der Spur als Beschreibungskategorie genutzt wurde. ${ }^{20}$ Diese Metapher erscheint mir hilfreich, um sich den Prozess der Bedeutungszuweisung $\mathrm{zu}$ vergegenwärtigen: Ausgehend von einem initialen Verstehensmoment kombiniert der Rezipient die weiteren Informationen sukzessive unter Anbindung an das Vorangegangene. Dadurch werden die Bedeutungen der Komponenten zusammengesetzt und bilden so eine zusammenhängende Einheit, die durch ein globales semantisches Feld, ${ }^{21}$ das Hans

18 Eine Konsequenz, die sich völlig stimmig zu den Prämissen und Schlussfolgerungen der IBE-Theorie (=Inference to Best Explanation) verhält. Vgl. dazu Peter Lipton, Inference to the best explanation. 2. Aufl. New York, London 2004.

19 Als Salienz verstehe ich gemäß Susan Fiske und Shelley Taylor „making a piece of information more noticeable, meaningful, or memorable to audiences. An increase in salience enhances the probability that receivers will perceive the information, discern meaning and thus process it, and store it in memory“ (Robert M. Entman, 'Framing: Toward Clarification of A Fractured Paradigm'. In: Journal of Communication 43 (1993), S. 51-58, hier S. 53.) Vgl. auch Susan T. Fiske und Shelley E. Taylor, Social Cognition. New York 1991, S. 246.

20 Vgl. Maximilian Scherner, 'Textverstehen als "Spurenlesen”'. In: Text und Grammatik. Hg. v. Peter Canisius u.a. Bochum 1994, S. 317-340; Monika Schwarz, 'Kohärenz - Auf den materiellen Spuren eines mentalen Phänomens'. In: Gesprochene Sprache - transdisziplinär. Festschrift für Gottfried Meinhold. Hg. v. Margret Bräunlich u.a. Frankfurt am Main 2001, S. 151-160.

21 Juri Michailowitsch Lotman, Die Struktur literarischer Texte. München 1972, S. 360. 
Hörmann Sinnkonstanz ${ }^{22}$ genannt hat, gekennzeichnet ist - sodass zum Beispiel die Genese einer in sich stimmigen erzählten Welt erst möglich und plausibel wird. Diese Spuren liegen in der konkreten Verwendungssituation für den Rezipienten nicht gleichmäßig ausgeprägt vor. So erscheinen einige (subjektiv) auffälliger und überdecken andere, die erst wieder an einer ganz anderen Stelle der Textur salient wirken und bei einer Relektüre unter anderen Gesichtspunkten deutlicher hervortreten könnten.

Bei der Rezeption von Romanen aus Schrift und Bild - illustriert, bebildert, bimodal, durchbildert, etc. - handelt es sich um einen Spezialfall des Textverstehens: um „semiotisch integriertes Gesamtverstehen“, ${ }^{23}$ in dessen Umfang die bisher beschriebenen Prozesse kombiniert Anwendung finden. Textverstehen zielt dabei als eine „Folge von perzeptiven und kognitiven Operationen [...] auf den Sprache[=Schrift]-Bild-Abgleich und eine kontextsensible Sinnzuschreibung“. ${ }^{24}$ Die Geschichte eines Romans, die Spezifizierung der Figuren(-konstellationen) und die Funktion des Erzählers werden aus sämtlichen Komponenten des literarischen Artefakts prozessiert.

Unter dieser Perspektive richte ich nun den Blick „,auf die binnentextuellen Verfahren der Erzeugung von Ambiguität als einem Doppelsystem einander gegenseitig ausschließender Deutungsmöglichkeiten in einem Text“. ${ }^{25}$ Eine Narration wird erst dann ambig, wenn der Rezipient

jeweils nur die eine oder die andere Sichtweise der Ereignisse akzeptieren [kann], wobei der Text selbst keinerlei Hinweis auf die Präferenz der einen oder der anderen Lesart gibt. Ein ambiguer Text erzeugt [...] somit stets eine unmögliche Situation für den Leser, indem er ihn mit verschiedenen Deutungsangeboten konfrontiert, die einander gegenseitig ausschließen [... . ${ }^{26}$

22 Hans Hörmann, Meinen und Verstehen. Grundzüge einer psychologischen Semantik. Frankfurt am Main 1976, S. 205, 208-212.

23 Werner Holly, 'Audiovisuelle Hermeneutik. Die Zeitgebundenheit des Bild-Verstehens am Beispiel der Medienberichterstattung'. In: Linguistische Hermeneutik. Theorie und Praxis des Verstehens und Interpretierens. Hg. v. Helmuth Feike und Angelika Linke. Tübingen 2007, S. 387-422, hier S. 390 .

24 Hartmut Stöckl, 'Sprache-Bild-Texte lesen. Bausteine zur Methodik einer Grundkompetenz'. In: Bildlinguistik. Hg. v. Hans-Joachim Diekmannshenke, Michael Klemm und Hartmut Stöckl. Berlin 2011, S. 43-70, hier S. 55.

25 Pinkas (vgl. Anm. 17), S. 39. Im Gegensatz dazu vertritt Marina Münkler ein weites Ambiguitätsverständnis, das auch widersprüchliches Figurenverhalten einschließt - dies wäre noch im Detail zu prüfen. Vgl. Marina Münkler, Narrative Ambiguität: Die Faustbücher des 16. bis 18. Jahrhunderts. Göttingen 2011.

26 Pinkas (vgl. Anm. 17), S. 39-40 (Hervorhebung NP). 
Die Herausforderung besteht somit nicht in der Vermeidung der Ambiguitätserfahrung, sondern in der Integration derselben in den eigentlichen Textsinn. Allgemeine Interpretationsoffenheit, die sich aus Merkmalen der Textur oder aber auch subjektiven Eigenschaften des Rezipienten speist, und der Spezialfall Ambiguität lassen sich demnach deutlich voneinander unterscheiden. Mit Blick auf analytische Präzision sollte Interpretationsoffenheit daher graduell im Rahmen eines Kontinuums verstanden werden. Ambiguität lässt sich demgegenüber durch die Analyse der intra- oder intertextuellen Verweissysteme einer Textur, mittels der zwei (oder mehr) äquivalent plausible Lesarten einer Textstelle und damit gegebenenfalls des gesamten Textes möglich werden, verorten. ${ }^{27}$

Das strukturbildende Moment der Wiederholung, für das Bildrepliken eine spezifische Form der Ausprägung darstellen, kann innerhalb narrativer Großformen eng mit der Etablierung bzw. Destabilisierung sinnkonstanter Entwürfe einer erzählten Welt verbunden sein. Ein Bild innerhalb eines Romans als die Wiederholung eines vorangehenden $z u$ verstehen, $d . h$. die erfolgte SegmentIteration zu erfassen, bedeutet, dass „die Wahrnehmung eines Elementes (A) ausdrücklich von dem Bewußtsein begleitet wird, es handele sich hier in der Tat um die Wiederholung eines Früheren (also um $\mathrm{A}^{0}$ oder genauer $\left.{ }^{0}[\mathrm{~A}]\right)^{\mathrm{c}} .{ }^{28}$ Das wiederholte Bild wird ebenso wie das sich wiederholende Bild damit $\mathrm{zu}$ einer intratextuellen Abbildung des jeweils anderen. In diesem engen Sinne stellt der erneute Verweis auf ein Motiv oder die Anspielung auf spezifisches textinternes oder -externes Wissen zwar eine Wiederaufnahme dar, genügt aber nicht der engen Identitätsbeziehung, die für eine Wiederholung konstitutiv ist. Eine Wiederholung wird als solche nur durch das Vorangehen bzw. das Nachfolgen von etwas Identischem ersichtlich, $\mathrm{A}$ und $\mathrm{A}^{0}$ sind nur in Relation zueinander wiederholt bzw. wiederholend.

27 Die Ambiguität eines Textes ist nicht immer eine im strengen Sinne narrative, wenngleich die Übergänge zwischen den unterschiedlichen Subklassen noch nicht ausreichend ausgeleuchtet wurden. Pinkas Studie zur Gestalt des phantastischen Films aus filmnarratologischer Sicht gründet ebenso wie Martínez' eher fiktionstheoretische Ausarbeitung Doppelte Welten auf Tzvetan Todorovs Ausführungen zum Phantastischen. Die Übergänge zwischen Narratologie im strengen Sinne und Fiktionstheorie erscheinen hier fließend - gleichwohl bleibt offen, wie sich die narrative Ambiguität einer Geschichte und die aus der Unklarheit, ob ein Text die Attribute fiktiv, faktual oder phantastisch verdient, resultierende Deutungsoffenheit zueinander verhalten. Vgl. Tzvetan Todorov, Introduction à la littérature fantastique. Paris 1970; Matias Martínez, Doppelte Welten. Struktur und Sinn zweideutigen Erzählens. Göttingen 1996; Pinkas (vgl. Anm. 17).

28 Eckhard Lobsien, Wörtlichkeit und Wiederholung: Phänomenologie poetischer Sprache. München 1995, S. 15-17. 
Insbesondere für die Struktur der Erzählung und die Gestalt der erzählten Welt hat eine solche Bezugnahme durch Iteration Konsequenzen. Der bis zu diesem Zeitpunkt der Textgenese sinnkonstante Entwurf der erzählten Welt gerät ins Stocken. Narrative Gewissheit, die sich zuvor etablierte, wird instabil:

Der Tendenz nach hebt jede Wiederholung die bis dato geleistete Synthesearbeit und die in ihr begründete und durch sie erworbene Sicherheit in der Lektüre wieder auf, und die lineare Sukzession, die Ausgedehntheit des Textes tritt wieder, wie zu Beginn einer jeden Lektüre, als Grundorientierung in Funktion. A und $\mathrm{A}^{0}$ stehen nebeneinander; ihre Wiederholung entautomatisiert die subjektive Aneignung des Textes [. . . ${ }^{29}$

Auf diese Weise kommt es zu einer Erhöhung der kognitiven Verarbeitungsleitung, die der Rezipient für die Genese eines Gesamttextes aufbringen muss. Der Herausforderung, die wiederholte Bildtextur im Rahmen des Schrift-Bild-Abgleichs in die Narration einzubinden, korrespondiert dabei zusätzlich die Integration der Wiederholung selbst. Die daraus gegebenenfalls resultierende Ambiguität dient hier als narratives Mittel, um neben einer allgemeinen Vagheit der Interpretationen distinkte, erzählte Welten einander gegenüber zu stellen. Diese können sich auf Ebene der Figuren, der Ereignisse, der Handlungen, des Modus, des Erzählers oder auch hinsichtlich der kausalen oder temporalen Ordnung derselben unterscheiden. Da Rezipienten in der Lage sind, unter Rückgriff auf unterschiedlichste epistemische Ressourcen (textinternes sowie textexternes Wissen) und unter Anwendung verschiedener kognitiver Kompetenzen, komplexe Bedeutungseinheiten zu konzeptualisieren, stellen semiotisch verschränkte Texturen somit ein erhebliches Potenzial bereit. Die Textgenese erfolgt auch hier sukzessive, aber nicht ohne Vorausdeutungen, Annahmen, Rückgriffe, Revisionen und Korrekturen - sie ist nicht an die gegenständliche Linearität der Textur gebunden. Epistemische Gewissheiten, die in der Lektüre über Identifikations-, Relationsund Schlussfolgerungsprozesse generiert werden, geraten so (wiederholt) instabil - so sehr, dass alles bis hin zur mimetischen Unentscheidbarkeit zu zerfallen droht. $^{30}$

29 Lobsien (vgl. Anm. 28), S. 10-11.

30 Vgl. Matias Martínez und Michael Scheffel, Einführung in die Erzähltheorie. 10. überarbeitete und aktualisierte Auflage. Köln 2016, S. 109-110. 


\section{Bildwiederholungen im Ritter Galmy II}

Das literarische Potenzial, das sich daraus ergibt, Bildwiederholungen in eine narrative Großform wie den Roman integrieren zu können, zeigt sich nun beispielsweise an der Konstruktion einer ambigen Narration. Hier markiert nicht etwa die Auflösung der gleichzeitigen Zweiwertigkeit, sondern die Synthese der irritierenden Simultaneität auf einer höheren Textebene den Fluchtpunkt. Statt $\mathrm{zu}$ fragen, welche Version die plausiblere ist, gilt es zu fragen, warum hier zwei oder mehrere Versionen in gegenseitiger Konkurrenz entworfen werden.

Dabei sind für die folgende Lesart mindestens die nachstehenden drei Prämissen zu nennen, deren Aussetzung zwar möglich ist, mir aber im Kontext literarischer Texturen wenig sinnvoll erscheint:

1. Soll nach narrativen Möglichkeiten gefragt werden, die sich aus der Bildwiederholung ergeben, so ist auszuschließen, dass die Doublette(n) entweder nicht wahrgenommen oder aber bewusst übergangen werden. Ersteres erscheint wahrnehmungspsychologisch mit Blick auf die Identitätsbeziehung der beiden Texturen zueinander unwahrscheinlich; ${ }^{31}$ letzteres bleibt dem Rezipienten überlassen, impliziert aber eine Bewertung der Iteration als versehentlich, fehlerhaft oder bedeutungslos für die Geschichte. ${ }^{32}$

2. Wenngleich eine Re-Semantisierung, also die Umdeutung einer bereits mit einer ersten Lesart versehenen Textur, zweifelsohne möglich ist, so wird dadurch die erste Lesart nicht etwa überschrieben und getilgt. Vielmehr existieren beide bzw. im Fall multipler Verwendung alle Lesarten simultan und distinkt fort, insbesondere dann, wenn eine die beiden Lesarten

31 Verwandt wären hier Überlegungen zur Funktionalen Gebundenheit, d. h. zur Unfähigkeit, ein Artefakt einer anderen Funktion zuzuführen.

32 Davon unbenommen ist die Hypothese, dass Bildwiederholungen auch eine ökonomische Attraktivität zukommt. Die Möglichkeit der mehrfachen Verwendung desselben Druckstocks beinhaltet gleichzeitig die Option, Kosten, wie sie z. B. durch die Anfertigung eines weiteren Druckstocks entstehen würden, zu sparen; vgl. Manuel Braun, 'Illustration, Dekoration und das allmähliche Verschwinden der Bilder aus dem Roman (1471-1700)'. In: Cognition and the Book. Typologies of Formal Organisation of Knowledge in the Printed Book of the Early Modern Period. Hg. v. Karl A. E. Enenkel und Wolfgang Neuber. Leiden 2005, S. 369-407, hier S. 382. Eine solche Hypothese bedarf jedoch der detaillierten Aufarbeitung der jeweiligen historischen Produktionsumstände, will sie nicht Spekulation bleiben, und läuft zudem beständig Gefahr, einer Selbstinszenierung der am Produktionsprozess beteiligten Akteure im Sinne der dissimulatio artis zu unterliegen. In jedem Fall - und zwar unabhängig davon, ob die Bildwiederholung literarisch oder merkantil motiviert Einzug in den Roman fand - ist sie Bestandteil der Textur und kann nicht einfach übergangen oder mit Verweis auf ihre Kontingenz bzw. als für die Interpretation irrelevant marginalisiert werden. 
rahmende Struktur existiert. Somit gilt diese Prämisse vornehmlich für den intratextuellen Wiederholungsfall und ist stark von weiteren Einflussgrößen (Distanz der wiederholten Elemente zueinander, Fortlauf der Lektüre, etc.) abhängig. Für die intertextuelle Wiederholung, wie sie z. B. zwischen Wickrams Ritter Galmy und weiteren Romanen, wie Der Jungen Knaben Spiegel (1554), zu beobachten ist, ${ }^{33}$ wäre diese Vorannahme daher differenzierter zu formulieren.

3. Da bei der Konstruktion der erzählten Welt des Ritter Galmy sowohl Schrift als auch Bild - im gegenseitigen Abgleich - einbezogen werden, können Bilder nicht als Zierrat marginalisiert werden, obgleich ihnen natürlich auch eine schmückende Funktion zukommt. Auf narrativer Ebene stellt sich die Frage, wer innerhalb der Logik des Romans Verantwortung für die Bilder und die Wiederholung derselben zeichnet. Dies ist nicht mit der Frage danach zu verwechseln, welche historische Produktionsinstanz die Verschränkung von Bildern und Schrift in diesem Fall initiiert bzw. umgesetzt hat.

Bevor nun ausgehend von diesen Prämissen und den vorangehenden Überlegungen die Auslegung der Bildwiederholung als literarischem Mittel und die damit einhergehenden narratologischen Konsequenzen fokussiert werden, subsumiere ich knapp die eingangs der Argumentation formulierte Lektüresituation:

Das erste Mal begegnet das Bild (Abb. 3) im vierten Kapitel des Romans und kann dort gemeinsam mit den schriftlich geschilderten Ereignissen der erzählten Welt zu einer plausiblen Lesart 1 kombiniert werden. Bei der zweiten Konfrontation mit dem Bild im 58. Kapitel - und dem damit verbundenen Wiedererkennen - wird diese etablierte Lesart 1 erneut aufgerufen, jedoch unmittelbar verworfen, da sie sich im Kontext der erzählten Ereignisse als inadäquat für diese Romanpassage erweist. Im Rahmen des erneut erforderlichen Verstehensprozesses erfolgt eine Re-Semantisierung der Textur an deren Ende eine Lesart 2 steht, die sich hinsichtlich zentraler Merkmale (z. B. Figurenidentität im Bild) von Lesart 1 unterscheidet. Innerhalb des Romans hat sich das Bild als ambige Textur erwiesen; die Lesarten 1 und 2 existieren simultan, distinkt und exklusiv fort.

Aus dieser Koinzidenz ergibt sich die Frage nach dem Verhältnis von Lesart 1 und Lesart 2 zueinander. Da es nach der Wiederholung unmöglich ist, die eine Bedeutung ohne die andere zu verstehen, gilt es, die in Schrift und

33 Im Roman Der Jungen Knaben Spiegel, der 15 Jahre nach der History von dem edlen und theüren Ritter Galmien erschienen ist, begegnen vier Bildtexturen erneut - dann jedoch singulär -, die bereits im Ritter Galmy mehrfach verwendet wurden. 


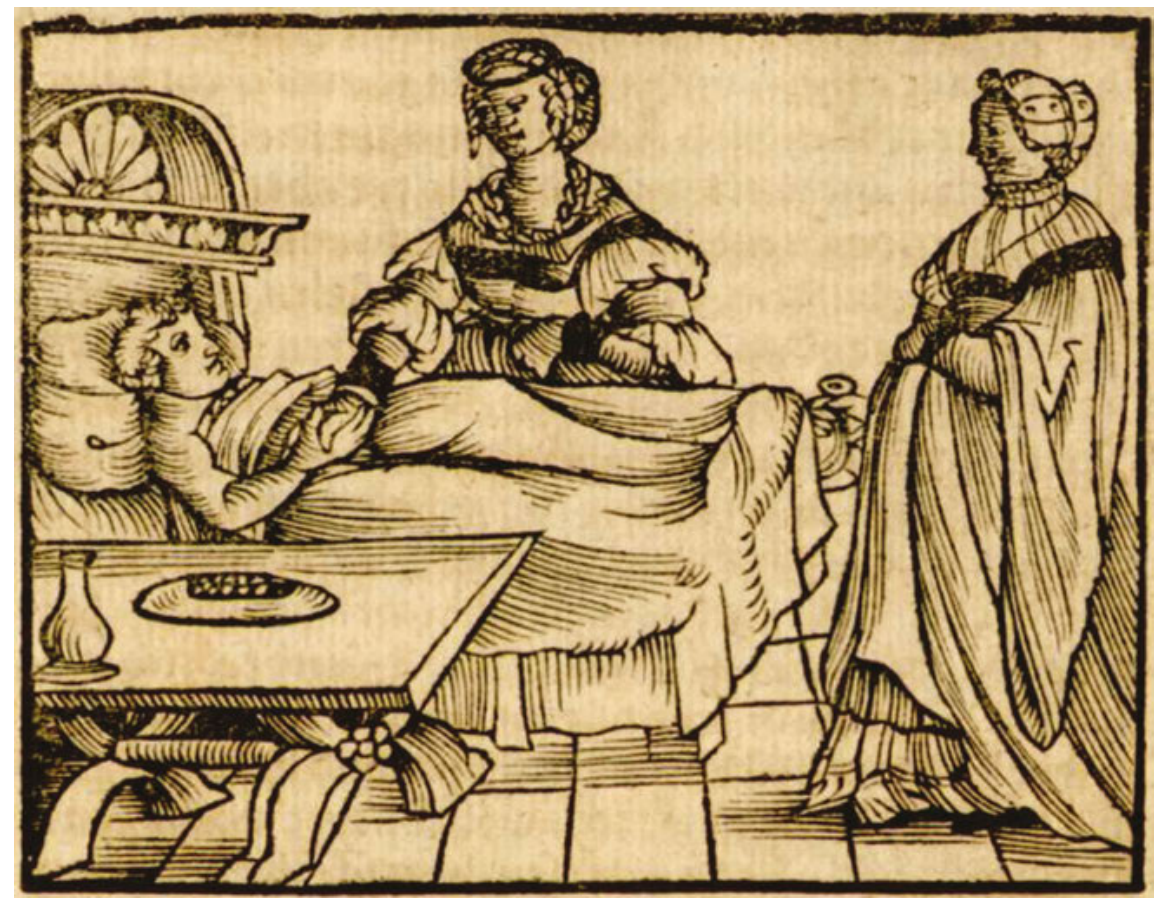

Abb. 3: Ritter Galmy (1539): fol. $9^{r}, 134^{r}$.

Bild vorliegenden Ereignisse des vierten und 58. Kapitels vor der Folie der weiteren bzw. vorangehenden Romanhandlung mit- und gegeneinander $\mathrm{zu}$ interpretieren:

Auf einer übergeordneten Ebene fällt schließlich eine strukturelle Ähnlichkeit der betreffenden Romanepisoden auf - eine Frau besucht einen im Bett liegenden Mann. Beide Situationen sind aus dieser allgemeinen Perspektive vergleichbar. Mit Ausnahme der vertauschten Männerfigur im Bett ist das im Bild dargestellte Figurenensemble möglicherweise identisch (die jeweiligen Zofen werden nicht eigens benannt) - so scheint es: Auffällig ist, dass die Krankheit des Herzogs ebenso unversehens vermittelt wird wie zuvor die Genesung des Ritters. Dem bildlichen Austausch der Männer korrespondiert zudem eine Permutation der Männer auf Handlungsebene. So wie der Herzog nämlich Galmys Platz im Krankenbett einnimmt, nimmt Galmy im unmittelbaren Fortlauf der Ereignisse den Platz des Herzogs im Bett der Herzogin und an der Spitze der Hofgesellschaft ein. Dieser chiastische Tausch rahmt die wechselseitige Liebessituation, denn um eine solche handelt es sich explizit, spricht doch 
die Herzogin in direkter Rede davon, dass sie Galmy „,von dem tag an liebhaben wil / in gleichem“ (fol. $135^{\mathrm{r}}$ ), wie er sie begehrt.

Platonisch und damit moralisch unzweifelhaft ist dieses Liebesverständnis - wenngleich der Erzähler dies mehrfach beteuert - zwischen Herrin und Vasall keineswegs, bereut sie doch erneut in Figurenrede ausdrücklich, dass sie nicht „,in eyner andern gestalt eüwers [= Galmys] leibs halben heimsuochen“ (fol. $8^{\mathrm{v}}$ ) kann und fordert von ihrem zukünftigen Geliebten, er solle es „mit gedult vertragen“ (fol. $8^{\mathrm{v}}$ ). Diese Geduld macht sich dann auf fol. $134^{\mathrm{r}}$ rasch bezahlt. Nachdem der Herzog, der dem Liebesglück im Wege stand, verstorben ist, überschlagen sich sogleich die Ereignisse: Bereits vier Seiten nach dem abrupten Tod des Herzogs schließt Galmy die Herzogin in seine Arme (s. Abb. 4). Dabei ist das Bild der Wiedervereinigung des Liebespaars sogar von dem Satz gerahmt: „So bald Galmy der Ritter von seinem gsellen vernan / das der Her[Abb. 4]-tzog tod was / sich von stund an bereyten thet" (fol. 135 $5^{\mathrm{v}}-136^{\mathrm{r}}$ ). Schließlich gipfelt die plötzlich rasant voran getriebene Handlung in der Hochzeit der beiden Liebenden. Im Anschluss erhält Galmy zudem den Titel eines bretonischen Herzogs. Obwohl der Schrifttext Galmys Ernennung zum neuen Herrscher der Bretagne nicht explizit problematisiert, wird doch betont, „das noch keyn Jar vschinen war / das der Hertzog gestorben was“ (fol. 138 ${ }^{\mathrm{r}}$ ).

Reflektiert man die Ereignisse unter Berücksichtigung dieser Aussage und vor dem Hintergrund der Parallelstruktur, die die Ambiguität der Schrift-BildTextur eröffnet, so wird - insbesondere vor dem Hintergrund der Entsprechung von liebeskrankem Ritter und sterbendem Herzog - eine Problematisierung der Situation ersichtlich: Auch wenn die erzählte Welt zeitlich nicht exakt zu verorten ist, lassen die geschilderten gesellschaftlichen Strukturen eine zeitliche Einordnung im Zeitraum vom 12. bis 13. Jahrhundert $\mathrm{zu}^{34} \mathrm{Zu}$ dieser Zeit setzte sich das gelehrte Römische Recht in der Bretagne zunehmend gegen das altgediente Gewohnheitsrecht mit seinen römischen, englischen und keltischen Einflüssen durch. Während das Englische Gewohnheitsrecht, das die Waliser und Inselkelten bei ihrem Exodus aus England besonders im 5. und 6. Jahrhundert mitbrachten, verlangt, dass Witwen „dem Tod des Ehemannes zwölf Monate lang unverheiratet blieben“, 35 da sie sonst die „Morgengabe und allen anderen

34 Von etwa 1341 an wird die gesamte Bretagne für mehrere Jahrzehnte bis 1379 vom Bretonischen Erbfolgekrieg erschüttert, von dessen destabilisierender Wirkung der Roman jedoch nicht berichtet. Außerdem ist die Blütezeit des Ritterstandes zu diesem Zeitpunkt bereits Vergangenheit. Die starken Landesherren und die deutlich ausgeprägte höfische Gesellschaft legen hochmittelalterliche Strukturen nah.

35 Jens Roehrkasten, 'Witwe, Englisches Recht'. In: LexMA, Band IX. Stuttgart, Weimar 1999, S. 279. 


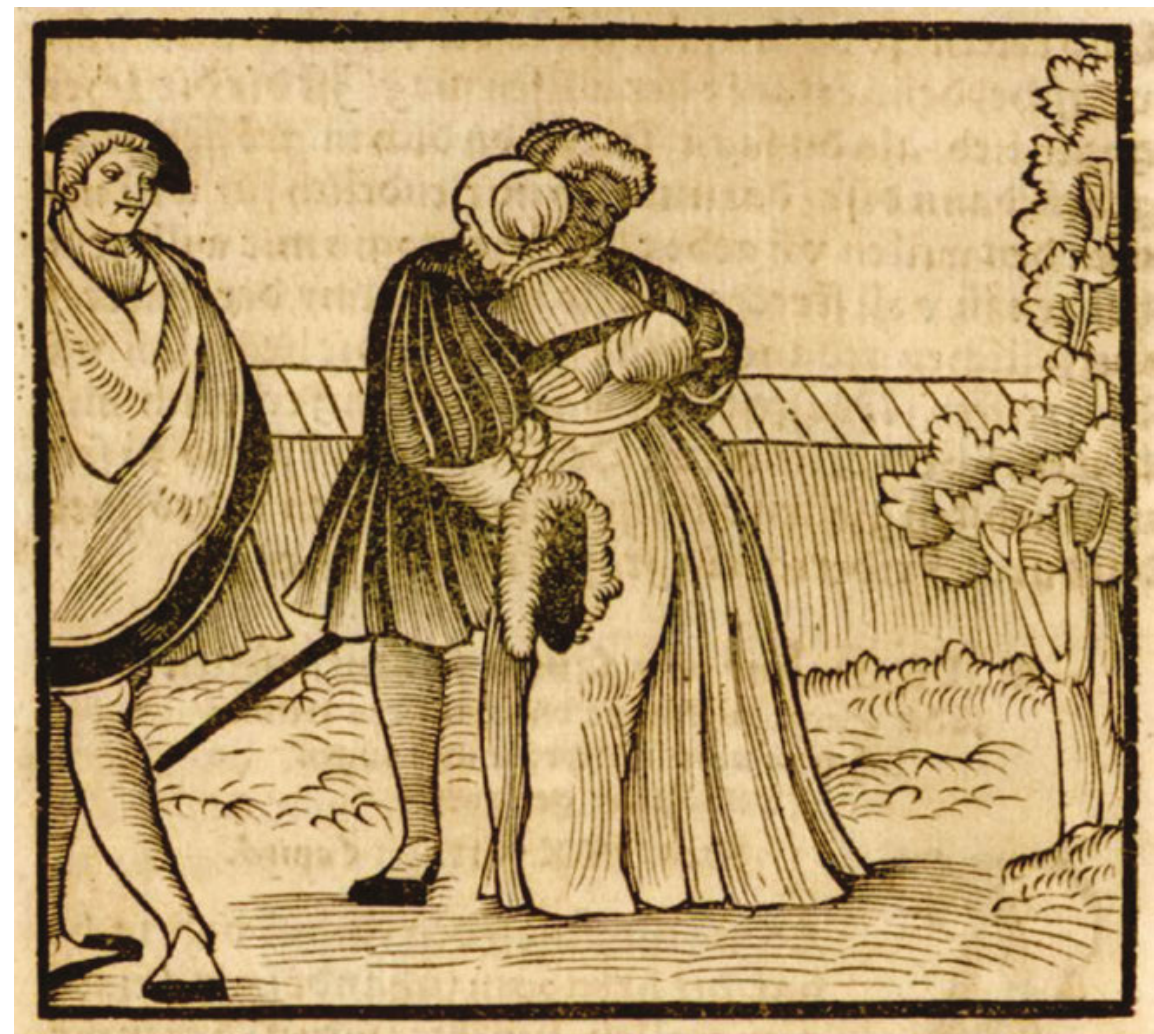

Abb. 4: Ritter Galmy (1539): fol. 136'.

durch den ersten Ehemann erhaltenen Besitz verlieren“36 sollten, fordert das Römische Recht - dessen Einfluss in der Bretagne bis ins 13. Jahrhundert stark bleibt - die vollständige und dauerhafte Infamie der Witwe, sollte diese neu heiraten (wollen). Konkret bedeutet dies den Verlust des gesellschaftlichen Rechtsschutzes und der Rechtsfähigkeit der Witwe. ${ }^{37}$ Demgegenüber schließt das Römisch-

36 Roehrkasten (vgl. Anm. 35).

37 Vgl. Irene Fuchs, Die Ehrenstrafen der Vergangenheit und Gegenwart. Diss. Köln 1928; Benno Löbmann, Der kanonische Infamiebegriff in seiner geschichtlichen Entwicklung: unter besonderer Berücksichtigung der Infamielehre des Franz Suarez. Leipzig 1956; Satu Lidman, Zum Spektakel und Abscheu: Schand- und Ehrenstrafen als Mittel öffentlicher Disziplinierung in München um 1600. Frankfurt am Main, Berlin, Bern 2008. 
Kanonische Recht, dessen Etablierung „in Nordfrankreich [...] schon im 13. Jh.“38 seinen Anfang nahm und „bereits Ende des 15. Jahrhunderts als abgeschlossen angesehen“39 werden kann, eine Wiederheirat grundsätzlich nicht mehr aus. Folglich lässt sich die erneute Heirat der Herzogin innerhalb des Trauerjahres nur mit den Vorgaben des Römisch-Kanonischen Rechts in Einklang bringen, da es innerhalb des Romans zu keiner unmittelbaren moralischen Ächtung der Herzogin kommt und sie auch nicht aller Titel und Besitztümer beraubt wird. Sie muss sich allerdings der „verwilligung aller Landtsherrn“ (fol. 137 ${ }^{\mathrm{V}}$ ) für die schnelle Hochzeit und den damit verbundenen Männertausch sicher sein.

Die Herzogin besteht hier offenbar einen moralischen Grenzgang, der zwar vom neu-etablierten Römisch-Kanonischen Recht gebilligt, aber deshalb gesellschaftlich noch lange nicht selbstverständlich wird. Somit ist die Ehe zwischen Galmy und der Herzogin nichts Widerrechtliches, taugt allerdings wohl kaum länger - trotz aller Beteuerungen des Erzählers, dass es sich bei der bretonischen Herzogin um ein Musterbild weiblicher Tugend handele - als sittsames Vorbild. Zwar ist während der gesamten Romanhandlung bis zum Tod des Herzogs präsent, dass die Herzogin sich von zwei Männern angezogen fühlt, doch zeigt die Bildreplik dies konzentriert an zwei zentralen Stellen auf - mit ihr erhält eine weitere Stimme Einzug in das Erzählgeflecht, die nicht identisch ist mit der des Erzählers.

Die Parallelkomposition der Ereignisse, die in der bildlichen Entsprechung und dem Austausch der Männerfigur an der Seite der Herzogin gipfelt, wird erst im Moment einer scheinbaren Redundanz, die durch die Replik in den Rezeptionsprozess eintritt, vor Augen geführt. Der strategische Einsatz der Möglichkeit, unterschiedliche Figuren der Geschichte den dargestellten Figuren im Bild zuzuordnen und die daraus resultierende Ambiguität der Bildelemente heben hier die Brisanz des Männertauschs, d. h. die Besetzung der Position an der Seite der Herzogin gleich durch zwei männliche Figuren der Handlung, auf eine Art und Weise hervor, die in einem Konkurrenz- bzw. Kooperationsverhältnis zur (ausschließlich schriftlich vermittelten,) wertenden Erzählerstimme steht.

Gleichzeitig - und das mag von systematischem Mehrwert sein - wird die Spannung zwischen Figuren- und Erzählerrede, die ohnehin im Roman angelegt ist, zusätzlich betont. Anstelle der anfangs stabilen Situation eines heterodiegetischen Erzählers (nullfokalisiert), der sich in Vorausdeutungen und Einblicke in das

38 Filippo Ranieri, 'Römisches Recht, Rezeption'. In: LexMA, Bd. VII. Stuttgart, Weimar 1999. S. 1015.

39 Ranieri (vgl. Anm. 38). 
Innenleben der Figuren $\mathrm{zu}$ erkennen gibt, erscheint hier eine homodiegetische, wertende Erzählsituation wahrscheinlicher. Inwieweit auf systematischer Ebene eine Korrelation zwischen dem Modus der Erzählung (insbesondere Distanz) und dem Vorhandensein von Bildrepliken als Stilelement zwischen Vorausdeutung und Rückblende existiert, wäre nun Gegenstand einer anderen Abhandlung.

\section{Bibliographie}

Benzig, Josef, Bibliographie strasbourgeoise. Bd. 1. Baden-Baden 1981.

Blazejewski, Susanne, Bild und Text - Photographie in autobiographischer Literatur: Marguerite Duras' 'L'amant' und Michael Ondaatjes 'Running in the Family'. Würzburg 2002.

Bolte, Johannes und Willy Scheel (Hgg.), Georg Wickrams Werke. Erster Band. Galmy Gabriotto. Tübingen 1901 (Bibliothek des literarischen Vereins in Stuttgart 222).

Braun, Manuel, 'Illustration, Dekoration und das allmähliche Verschwinden der Bilder aus dem Roman (1471-1700)'. In: Cognition and the Book. Typologies of Formal Organisation of Knowledge in the Printed Book of the Early Modern Period. Hg. v. Karl A. E. Enenkel und Wolfgang Neuber. Leiden 2005, S. 369-407.

Drittenbass, Catherine und André Schnyder (Hgg.), Eulenspiegel trifft Melusine. Der frühneuhochdeutsche Prosaroman im Licht neuer Forschungen und Methoden. Amsterdam, New York 2010.

Entman, Robert M., 'Framing: Toward Clarification of A Fractured Paradigm'. In: Journal of Communication 43 (1993), S. 51-58.

Fischer, Hubertus, 'Wickrams Bilderwelt. Vorläufige Bemerkungen'. In: Vergessene Texte Verstellte Blicke. Neue Perspektiven der Wickram-Forschung. Hg. v. Maria Elisabeth Müller und Michael Mecklenburg. Frankfurt am Main 2007, S. 199-214.

Fiske, Susan T. und Shelley E. Taylor, Social Cognition. New York 1991.

Fix, Ulla und Hans Wellmann, 'Sprachtexte - Bildtexte. Bemerkungen zum Symposion Bild im Text - Text und Bild vom 6.-8. April 2000 in Leipzig'. In: Bild im Text, Text im Bild. Hg. v. Ulla Fix und Hans Wellmann. Heidelberg 2000, S. XI-XVII.

Fuchs, Irene, Die Ehrenstrafen der Vergangenheit und Gegenwart. [Diss.] Köln 1928.

Gotzkowsky, Bodo, 'Volksbücher'. Prosaromane, Renaissancenovellen, Versdichtungen und Schwankbücher. Bibliographie der Deutschen Drucke. Teil I: Drucke des 15. Und 16. Jahrhunderts. Baden-Baden 1991.

Gotzkowsky, Bodo, Die Buchholzschnitte Hans Brosamers zu den Frankfurter „Volksbuch“Ausgaben und ihre Wiederverwendungen. Baden-Baden 2002.

Holly, Werner, 'Audiovisuelle Hermeneutik. Die Zeitgebundenheit des Bild-Verstehens am Beispiel der Medienberichterstattung'. In: Linguistische Hermeneutik. Theorie und Praxis des Verstehens und Interpretierens. Hg. v. Helmuth Feike und Angelika Linke. Tübingen 2007, S. 387-422.

Hörmann, Hans, Meinen und Verstehen. Grundzüge einer psychologischen Semantik. Frankfurt am Main 1976.

Kartschoke, Dieter, 'Ritter Galmy uß Schottenland und Jörg Wickram aus Colmar'. In: Daphnis 31 (2002), S. 469-489. 
Keary, Mae, ‘Key Technologies - Chasing the Gap’. In: Aslib Proceedings 43/5 (1991),

S. 161-172.

Knape, Joachim, 'Mnemonik, Bildbuch und Emblematik im Zeitalter Sebastian Brants (Brant, Schwarzenberg, Alciati)'. In: Mnemosyne. Festschrift für Manfred Lurker zum 60. Geburtstag. Hg. v. Werner Bies und Hermann Jung. Baden-Baden 1988, S. 133-178.

Kuch, Raphael, Intermediales Erzählen im frühneuzeitlichen illustrierten Roman. Köln, Weimar, Wien 2014.

Lidman, Satu, Zum Spektakel und Abscheu: Schand- und Ehrenstrafen als Mittel öffentlicher Disziplinierung in München um 1600. Frankfurt am Main, Berlin, Bern 2008.

Lipton, Peter, Inference to the best explanation. 2. Aufl. New York, London 2004.

Löbmann, Benno, Der kanonische Infamiebegriff in seiner geschichtlichen Entwicklung: unter besonderer Berücksichtigung der Infamielehre des Franz Suarez. Leipzig 1956.

Lobsien, Eckhard, Wörtlichkeit und Wiederholung: Phänomenologie poetischer Sprache. München 1995.

Lotman, Juri Michailowitsch, Die Struktur literarischer Texte. München 1972.

Martínez, Matias, Doppelte Welten. Struktur und Sinn zweideutigen Erzählens. Göttingen 1996.

Martínez, Matias und Michael Scheffel, Einführung in die Erzähltheorie. 10. überarbeitete und aktualisierte Auflage. Köln 2016.

Münkler, Marina, Narrative Ambiguität: Die Faustbücher des 16. bis 18. Jahrhunderts. Göttingen 2011.

Pinkas, Claudia, Der phantastische Film. Instabile Narration und die Narration der Instabilität. Berlin, New York 2010.

Potysch, Nicolas, Wiederholt doppeldeutig in Bild und Schrift - Ambiguität im durchbilderten Roman. Hannover 2018.

Ranieri, Filippo, ‘Römisches Recht, Rezeption'. In: LexMA. Bd. VII. Stuttgart, Weimar 1999. S. 1015.

Roehrkasten, Jens, 'Witwe, Englisches Recht'. In: LexMA. Bd. IX. Stuttgart, Weimar 1999. S. 279.

Scherner, Maximilian, ‘Textverstehen als “Spurenlesen”'. In: Text und Grammatik. Hg. v. Peter Canisius u.a. Bochum 1994. S. 317-340.

Schmidt, Peter, 'Literat und "selbstgewachsener Moler". Jörg Wickram und der illustrierte Roman der Frühen Neuzeit'. In: Künstler und Literat. Schrift- und Buchkultur in der europäischen Renaissance. Hg. v. Bodo Guthmüller, Berndt Hamm und Andreas Tönnesmann. Wiesbaden 2006, S. 143-194.

Schwarz, Monika, 'Kohärenz - Auf den materiellen Spuren eines mentalen Phänomens'. In: Gesprochene Sprache - transdisziplinär. Festschrift für Gottfried Meinhold. Hg. v. Margret Bräunlich u.a. Frankfurt am Main 2001, S. 151-160.

Stöckl, Hartmut, Die Sprache im Bild, das Bild in der Sprache: Zur Verknüpfung von Sprache und Bild im massenmedialen Text. Konzepte, Theorien, Analysemethoden. Berlin, New York 2004.

Stöckl, Hartmut, 'Sprache-Bild-Texte lesen. Bausteine zur Methodik einer Grundkompetenz'. In: Bildlinguistik. Hg. v. Hans-Joachim Diekmannshenke, Michael Klemm und Hartmut Stöckl. Berlin 2011, S. 43-70.

Susanka, Thomas M., Foto/grafie. Zur Rhetorik von Medium und Bild. Mit einer Fallstudie zu James Nachtwey. Berlin 2015.

Todorov, Tzvetan, Introduction à la littérature fantastique. Paris 1970. 
